\title{
CALPÚN-INIA, CULTIVAR DE LENTEJA (Lens culinaris Medik.) DE GRANO GRANDE Y RESISTENTE A ROYA
}

\section{Calpún-INIA, a large seed size and rust-resistant lentil (Lens culinaris Medik.) cultivar}

\author{
Enrique Peñaloza ${ }^{1}$ *, Juan Tay U. $^{2}$ y Andrés France I. $^{2}$
}

\begin{abstract}
A B S T R A C T
Calpún-INIA is a rust (Uromyces fabae Pers.) resistant lentil (Lens culinaris Medik.) cultivar that was developed by the former Food Legume Program of the National Institute of Agricultural Research (INIA), Chile. It is derived from crosses between the Chilean rust-susceptible Araucana-INIA and the Canadian rustresistant Laird cultivars, using earliness, AraucanaINIA's type of plant architecture, seed size, seed yield and rust resistance as selection criteria. Calpún-INIA belongs to the large seeded cultivars (macrosperma type), averaging 8 g per 100 seeds, and $>65 \%$ of seeds retained in a $7 \mathrm{~mm}$ sieve. The seed is partially flattened, with pale green to grey background and pale yellow cotyledons. Averaged across years, the seed yield of Calpún-INIA was $17 \%$ lower than AraucanaINIA's in rust-free environments, whereas CalpúnINIA out-yielded Araucana-INIA by 60 to $95 \%$ in environments where this disease is the main constraint for lentil production. Calpún-INIA is the first lentil cultivar released by INIA, combining high yield and large seed size with rust resistance.
\end{abstract}

Key words: Lens culinaris, Araucana-INIA, Laird, Uromyces fabae.

\section{R E S U M E N}

Calpún-INIA es un cultivar de lenteja (Lens culinaris Medik.) resistente a la roya (Uromyces fabae Pers.), desarrollado por el ex Programa de Leguminosas de Grano del Instituto Nacional de Investigaciones Agropecuarias (INIA), Chile. Se origina de un cruzamiento entre el cultivar chileno Araucana-INIA susceptible a la roya y el cultivar canadiense Laird resistente a roya, utilizando precocidad, arquitectura de planta del tipo Araucana-INIA, tamaño de grano, rendimiento de grano y resistencia a roya como criterios de selección. Calpún-INIA pertenece a los cultivares de tamaño de grano grande (tipo macrosperma), con peso promedio de 8 g por 100 granos, y con tamaño de grano de $7 \mathrm{~mm}$ superior a $65 \%$. La semilla es parcialmente aplanada, de color verde claro a gris y de cotiledones amarillo pálidos. En promedio de temporadas, el rendimiento en grano de Calpún-INIA fue 17\% inferior a Araucana-INIA, en evaluaciones realizadas en ambientes libres de roya. En tanto, su rendimiento supera a Araucana-INIA entre 60 a 95\%, en ambientes donde la enfermedad es la principal limitante de producción. Calpún-INIA es el primer cultivar de lenteja desarrollado por INIA que combina alto rendimiento y calibre de grano, con resistencia a roya.

Palabras clave: Lens culinaris, Araucana-INIA, Laird, Uromyces fabae.

\footnotetext{
${ }^{1}$ Instituto de Investigaciones Agropecuarias, Centro Regional de Investigación Carillanca, Casilla 58-D, Temuco, Chile. E-mail: epenaloz@inia.cl *Autor para correspondencia.

${ }^{2}$ Instituto de Investigaciones Agropecuarias, Centro Regional de Investigación Quilamapu, Casilla 246, Chillán, Chile. Recibido: 29 de mayo de 2006. Aceptado: 30 de agosto de 2006.
} 


\section{INTRODUCCIÓN}

La lenteja (Lens culinaris Medik.) es una leguminosa de grano tradicionalmente cultivada en los secanos costero e interior de la zona central y centro-sur de Chile. Su superficie de siembra superó las 50.000 ha en la década de los ochenta, cuando una alta proporción de la producción se exportaba principalmente a mercados sudamericanos. Esta superficie se ha reducido sostenidamente durante los últimos años para representar cerca de 1.300 ha en la actualidad. Como principales responsables de esta menor superficie aparece la importación de lenteja desde Canadá, la escasa incorporación de tecnología suficiente para aumentar su competitividad, y la incidencia de roya (Uromyces fabae Pers.).

La roya representa una de las principales limitantes de la producción de lenteja en siembras de invierno en el secano costero y en siembras de primavera en suelos de vegas de los secanos costero e interior del centro-sur de Chile. Para enfrentar este problema, en la década del setenta se introdujeron al país cultivares de lenteja resistentes a roya, como Penzeskaja 14 y Laird (Tay et al., 2001a). Sin embargo, el tamaño de grano de estos materiales era muy inferior a la lenteja chilena, por lo que no se ajustaron a las normas de calidad requeridas por los mercados internacionales. Aun cuando el posterior desarrollo de Araucana-INIA (Tay et al., 1981) y Super Araucana-INIA (Tay et al., 2001b) permitió aumentar el tamaño del grano, ambos cultivares no resolvieron el problema en los secanos costero e interior debido a su extrema susceptibilidad a la roya y al elevado costo de las prácticas de control químico.

La investigación realizada por el Instituto Nacional de Investigaciones Agropecuarias (INIA) durante el decenio 1980-1990 permitió desarrollar el cv. Calpún-INIA, que compatibiliza alto calibre de grano con la resistencia a la roya. Esta variedad ofrece la posibilidad de recuperar áreas tradicionales de cultivo donde esta enfermedad representa la principal limitante de producción.

\section{ORIGEN Y PEDIGRÍ}

Calpún-INIA es un cultivar obtenido del cruzamiento entre Araucana-INIA $\times$ Laird, utilizando arquitectura de planta del tipo Araucana-INIA, precocidad, rendimiento, calibre de grano y resistencia a roya como criterios de selección. En la etapa F7 este material se multiplicó en una zona con alta incidencia de roya, para realizar selecciones de planta individual bajo los criterios antes mencionados. A partir de la temporada 1993-1994 se incorporó a ensayos de rendimiento en Temuco (IX Región), Chanco y Curepto (VII Región) con la sinonimia de LC-84-9000-23-24. Esta línea destacó por su resistencia a roya heredada del cv. Laird, y su arquitectura de planta y precocidad característicos de Araucana-INIA. Un atributo particular de CalpúnINIA es su tamaño y calibre de grano, superior a ambos progenitores.

\section{DESCRIPCIÓN MORFOLÓGICA}

La planta es de crecimiento indeterminado, con altura promedio variable entre 30 y $50 \mathrm{~cm}$ dependiendo del ambiente de producción. Las flores son de color blanco, con venas de color azul en el estandarte, desarrollándose usualmente una vaina por pedúnculo, con un promedio de un grano por vaina. El grano es parcialmente aplanado de cutícula verde claro a gris y cotiledones amarillo claros, con un peso promedio de $8 \mathrm{~g}$ por 100 semillas.

\section{CARACTERÍSTICAS FITOPATOLÓGICAS}

Calpún-INIA presenta resistencia a la roya, demostrada en siembras de primavera en el secano costero, donde el agente causal de la enfermedad encuentra condiciones de humedad y temperatura óptimas para su desarrollo y diseminación. Evaluaciones realizadas en siembras de otoño indican que Calpún-INIA es susceptible al tizón de la lenteja ( $A s$ cochyta lentis Vassil), enfermedad que puede adquirir relevancia en siembras tempranas o en primaveras lluviosas. Aun cuando el tizón de la lenteja no necesariamente afecta el rendimiento, su incidencia adquiere importancia comercial debido a que los síntomas de la enfermedad se presentan en la superficie del grano causando un deterioro del precio.

\section{ZONA DE CULTIVO, DOSIS DE SEMILLA Y FECHA DE SIEMBRA}

Calpún-INIA tiene requerimientos de clima y suelo similares a Araucana-INIA y Super AraucanaINIA. Se recomienda para siembras de primavera en zonas con incidencia endémica de roya, como el secano costero entre la VII y VIII Regiones del país. La densidad de siembra sugerida corresponde a 70 a 80 plantas $\mathrm{m}^{-2}$, equivalentes a dosis de semilla entre 70 y $80 \mathrm{~kg} \mathrm{ha}^{-1}$. 


\section{RENDIMIENTO Y CALIBRE DE GRANO}

\section{Experimentos}

En promedio de cuatro temporadas, el rendimiento en grano de Calpún-INIA $\left(1.466 \mathrm{~kg} \mathrm{ha}^{-1}\right)$ fue $17 \%$ inferior a Araucana-INIA (1.770 $\left.\mathrm{kg} \mathrm{ha}^{-1}\right)$, en evaluaciones realizadas en un ambiente sin incidencia de roya (Temuco, IX Región). El calibre de grano, en tanto, es significativamente superior a Araucana-INIA y similar a Super Araucana-INIA (Cuadro 1). En presencia de roya, Calpún-INIA mantiene su rendimiento y calibre de grano característico. En estos ambientes, el rendimiento de Calpún-INIA fue entre 60 y $95 \%$ superior a los cultivares susceptibles Araucana-INIA y Super Araucana-INIA, respectivamente (Cuadro 2).

\section{Validación}

Calpún-INIA se validó en predios de agricultores, a través de programas de transferencia tecnológica financiados por el Fondo de Desarrollo Regional de la Región del Maule. Estas experiencias se realizaron en siembras de primavera, en suelos de vega de la localidad de Chanco (VII Región). El rendimiento en grano del cv. Calpún-INIA (identificado por los agricultores como variedad RR-INIA) fluctuó entre 1.100 a $1.300 \mathrm{~kg} \mathrm{ha}^{-1}$, y el rendimiento del calibre $7 \mathrm{~mm}$ entre 65 y $82 \%$ del grano cosechado. En estas siembras comerciales la incidencia de roya se calificó con nota 3 , en la escala convencional de 1 a 9 (Tay et al., 2000).

Cuadro 1. Rendimiento $\left(\mathrm{kg} \mathrm{ha}^{-1}\right)$ y calibre $7 \mathrm{~mm}(\%)$ de cultivares de lenteja evaluados durante cuatro temporadas en un ambiente libre de roya de la IX Región (Temuco).

Table 1. Seed yield $\left(\mathrm{kg} \mathrm{ha}^{-1}\right)$ and $7-\mathrm{mm}$ seed caliber $(\%)$ of lentil cultivars evaluated through four growing seasons in a rust-free environment of the IX Region (Temuco).

\begin{tabular}{|c|c|c|c|c|c|c|c|c|}
\hline \multirow[t]{2}{*}{ Cultivares } & \multicolumn{4}{|c|}{ Rendimiento } & \multicolumn{4}{|c|}{ Calibre 7 mm ${ }^{(1)}$} \\
\hline & 1993-1994 & 1994-1995 & 1995-1996 & 1996-1997 & 1993-1994 & 1994-1995 & 1995-1996 & $1996-1997$ \\
\hline Calpún-INIA & 1.403 & 1.903 & 1.164 & 1.392 & 70,0 & 73,2 & 90,0 & 84,0 \\
\hline Araucana-INIA & 1.645 & 2.278 & 1.452 & 1.703 & 33,3 & 42,2 & 78,0 & 60,9 \\
\hline Laird & 676 & 1.630 & 920 & 770 & 2,8 & 4,8 & 8,6 & 6,5 \\
\hline Super Araucana-INIA & s.i. & s.i. & 1.370 & 1.828 & s.i. & s.i. & 88,6 & 81,8 \\
\hline DMS (5\%) & 600 & n.s. & 261 & 460 & 8,5 & 10,5 & 5,0 & 3,9 \\
\hline CV (\%) & 16,0 & 16,1 & 11,7 & 14,4 & 12,0 & 14,5 & 6,0 & 3,1 \\
\hline
\end{tabular}

(1) Valores transformados a arcoseno $\sqrt{ } \mathrm{x}$ previo al análisis estadístico.

s.i.: Sin información; n.s.: No significativo (P > 0,05); DMS: diferencia mínima significativa; CV: coeficiente de variación.

Cuadro 2. Rendimiento $\left(\mathrm{kg} \mathrm{ha}^{-1}\right)$, calibre $7 \mathrm{~mm}(\%)$ y nota de roya de cultivares de lenteja evaluados en dos ambientes con alta incidencia de roya de la VII Región (Chanco, 1994-1995 y Curepto, 1997-1998).

Table 2. Seed yield ( $\left.\mathrm{kg} \mathrm{ha}^{-1}\right), 7-\mathrm{mm}$ seed caliber (\%) and rust score of lentil cultivars evaluated in two high rustprone environments of the VII Region (Chanco, 1994-1995 and Curepto, 1997-1998).

\begin{tabular}{lcccccccc}
\hline \multirow{2}{*}{ Cultivares } & \multicolumn{2}{c}{ Rendimiento } & & \multicolumn{2}{c}{ Calibre $\mathbf{7 ~ m ~}$} & & \multicolumn{2}{c}{ Nota roya $^{(\mathbf{1})}$} \\
\cline { 2 - 3 } & $\mathbf{1 9 9 4 - 1 9 9 5}$ & $\mathbf{1 9 9 7 - 1 9 9 8}$ & & $\mathbf{1 9 9 4 - 1 9 9 5}$ & $\mathbf{1 9 9 7 - 1 9 9 8}$ & & $\mathbf{1 9 9 4 - 1 9 9 5}$ & $\mathbf{1 9 9 7 - 1 9 9 8}$ \\
\hline Calpún-INIA & 1.390 & 1.550 & & 8,9 & 82,0 & & 2,5 & 4,0 \\
Araucana-INIA & 580 & 80 & & 12,6 & 2,0 & & 8,0 & 9,0 \\
Laird & 670 & s.i. & & 0,2 & s.i. & & 2,0 & s.i. \\
Super Araucana-INIA & s.i. & 60 & & s.i. & 3,0 & & s.i. & 9,0 \\
DMS (5\%) & 530 & 360 & & 5,5 & 7,3 & & & \\
CV (\%) & 18,4 & 12,6 & & 9,0 & 11,5 & & & \\
\hline
\end{tabular}

(1) Incidencia de roya evaluada en escala de 1 a 9 ( 1 = planta sin síntomas; 9 = planta muerta $)($ Tay et al., 2000). s.i.: Sin información; DMS: diferencia mínima significativa; CV: coeficiente de variación. 


\section{CALIDAD}

La calidad comercial de la lenteja está determinada fundamentalmente por el color y calibre del grano. Calpún-INIA cumple con estos requisitos, representando en la actualidad la primera variedad de lenteja de grano grande con resistencia a roya desarrollada en el país. Antecedentes adicionales indican que este cultivar se caracteriza por un contenido de proteínas de $22 \%$, un tiempo de cocción de 18-22 min después de $8 \mathrm{~h}$ de hidratación (remojo), y sabor y textura similar a los cultivares Araucana-INIA y Super Araucana-INIA.

\section{LITERATURA CITADA}

Tay,, J., M. Paredes, y V. Kramm. 1981. Araucana-INIA, variedad de lenteja de grano grande. Agric. Téc. (Chile) 41:170.

Tay, J., A. France, M. Gerding, V. Kramm, y R. Velasco. 2000. Manual de leguminosas de grano y hortícolas para el Secano Costero de la Región del Maule. Boletín INIA N $\mathrm{N}^{\circ} 40.94$ p. Instituto de Investigaciones Agropecuarias, Centro Regional de Investigación Quilamapu, Chillán, Chile.
Tay, J., E. Peñaloza, and A. France. 2001a. Breeding for resistance to lentil rust in Chile. p. 243. In Proceedings of the $4^{\text {th }}$ European Conference on Grain Legumes. Towards the sustainable production of healthy food, feed and novel products. European Association for Grain Legume Research, Cracovia, Poland.

Tay, J., A. France, y M. Paredes. 2001b. Super AraucanaINIA: una nueva variedad de lenteja (Lens culinaris Med.) chilena de grano grande. Agric. Téc. (Chile) 61:385-389. 